\title{
EL SIMBOLISMO DE LA TEORÍA DE LA RELATIVIDAD Y LA DURACIÓN COMO MULTI-TEMPORALIDAD
}

\author{
István Berszan \\ Universidad Babes, - Bolyai (Cluj)
}

En una famosa obra llamada Durée et simultanéité ([1922] 1923), Henri Bergson se propone un intento filosófico de la teoría de la relatividad elaborada por el físico Einstein. El filósofo considera inevitable semejante análisis, porque rechaza la posibilidad de la interferencia directa entre física y metafísica. En el prefacio del libro se critica cualquier intento de aceptar la física de Einstein como una filosofía. El iniciador del concepto de duración (concepto que puede ser compatible con un tiempo que lo contiene todo) examina en la teoría de la relatividad las paradojas temporales: los tiempos múltiples que no corren de la misma manera, la simultaneidad que se transforma en sucesión, y la sucesión que se transforma en simultaneidad. Lo que va a ser importante desde nuestro punto de vista no es la rehabilitación del tiempo real, sino la negación bergsoniana de la posibilidad de sustitución entre filósofo y físico. 
La teoría de la relatividad ha sido creada conforme a la exigencia científica de establecer las leyes de la física (concretamente de la electromagnética) de tal manera que ellas sean válidas para cualquier punto de vista de cualquier observador. La simultaneidad de un sistema $R$, observada desde el sistema $R$ que tiene una velocidad $v$ en relación con $R$, se transforma en sucesión por esta exigencia. También por ella el tiempo se debe dilatar, y achicar las distancias del sistema en movimiento. Así los universos, descritos desde distintas perspectivas, se organizan en base a las mismas relaciones, y con este resultado el físico está contento, porque está interesado sólo en las relaciones de los datos de medida. El filósofo, sin embargo, está interesado en «le concret, tout chargé de qualité» (Bergson, [1922]1923: 87) ${ }^{1}$, es decir, lo que dura y se mantiene en los intervalos de los datos de medida. Por eso, para él no son obligatoriamente idénticos los fenómenos que, en base a los datos de medida, respectivamente en base a la relación de estos datos con otros, pueden ser situados a la derecha, respectivamente a la izquierda de una ecuación matemática. Entre los tiempos posibles matemáticamente el filósofo de Bergson acepta solamente uno como tiempo real, todos los demás son convencional-simbólicos o imaginarios. En otras palabras, si en una continuidad espacio-temporal cuadridimensional es correcto suponer que los fenómenos como cantidades se pueden medir, filosóficamente hablando, esta suposición es un grave error producido por la espacialización del tiempo.

La problemática de la multiplicación del tiempo puede ser ilustrada por el experimento Michelson-Morley, en su fórmula en la que, siguiendo a Einstein, excluimos la existencia del éter estático.

En un sistema $R$ un rayo de luz atraviesa una pantalla de vidrio inclinada a 45 grados, y de esta manera se divide en dos. Uno de ellos avanza en la dirección del rayo de origen como una continuación de éste, «su pareja» en cambio se refleja desde la superficie de la pantalla en una dirección perpendicular sobre la línea seguida por el primero. Los dos rayos se encuentran con un espejo perpendicular sobre su dirección; los espejos están situados a la misma distancia del punto donde el rayo de origen se ha dividido; de esta forma los espejos reflejan los rayos sobre las mismas líneas a lo largo de las cuales éstos han llegado. El rayo que en el momento de la división del rayo de origen ha variado 90 grados, al volver a la pantalla de vidrio se va a dividir de

1 Las citas pertenecen a la edición Henri Bergson (1923): Durée et simultanéité. A propos de la Théorie d'Einstein, Paris: Librairie Félix Alcan. 
nuevo, de tal manera que una de sus prolongaciones avance en la dirección de la reflexión precedente, dirección en la que circula aquella continuación del rayo reflejado por el otro espejo que varía 90 grados de la superficie de la pantalla de vidrio. Si ponemos una lupa sobre esta línea, orientada hacia el punto de reflexión de la pantalla de vidrio, podemos observar las rayas de interferencia que aparecen después de la superposición de los rayos (véase la figura).

Si en un medio en el que la luz se desplaza en todas las direcciones con la misma velocidad, el sistema Michelson-Morley es estático, entonces el tiempo necesario para el desplazamiento de la luz de la pantalla de vidrio hasta uno de los espejos y al revés es igual al tiempo del desplazamiento de la luz de la pantalla de vidrio hasta el otro espejo y al revés:

$$
\text { espejo }
$$

fuente de luz

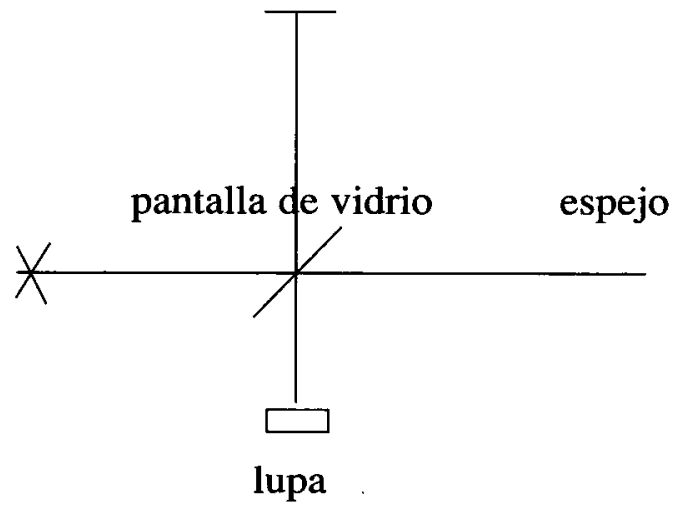

Pero si tenemos en cuenta que el aparato se mueve al mismo tiempo que la Tierra (supongamos que, durante la medición, a una velocidad constante sobre una línea recta y en la dirección en la que aquella continuación del rayo de origen que atraviesa la pantalla de vidrio), entonces la luz no debería «comportarse» igual sobre el brazo del aparato que apunta en la dirección del movimiento que sobre el brazo que es perpendicular sobre esta dirección. Porque sobre el brazo perpendicular el desplazamiento ida y vuelta del rayo no se ve influido por el movimiento del sistema ni en cuanto a las relaciones entre las dos distancias recorridas, ni en cuanto al tiempo necesario para recorrerlas: por tanto las dos distancias, es decir, los tiempos 
medidos en las dos direcciones, quedan iguales. Sin embargo, sobre el brazo que indica la dirección del movimiento de la Tierra, el camino de la pantalla de vidrio hasta el espejo no es igual al camino inverso $\mathrm{y}$, por consiguiente, tampoco el tiempo necesario para recorrer estas distancias, ya que durante el desplazamiento de la luz hacia el espejo el espejo rehuye la luz, y a la vuelta, la luz es recibida por la pantalla. De aquí resulta que en el caso de la inversión de los brazos, las rayas de interferencia deberían cambiar. Pero esto no pasa, todo se desarrolla como si la Tierra no se moviera. Y no vale la pena repetir el experimento en distintos periodos del año, es decir, en el caso de las diferentes velocidades de la Tierra, el resultado es el mismo. Lorentz ha explicado esto por el achicamiento de la distancia en la dirección del movimiento del sistema y por la dilatación del tiempo del movimiento, después de lo cual las simultaneidades se alejan. Ha calculado las diferencias por medio de ecuaciones de transformación que, desde entonces, llevan el nombre del físico.

La teoría de Einstein excluye la existencia de un sistema privilegiado que se encuentra en reposo absoluto, por eso, el movimiento de la Tierra no se relaciona con un éter fijo, sino, por ejemplo, con el Sol que, en relación con la Tierra, también se encuentra en movimiento, y es elegido como sistema de referencia de manera arbitraria. Aunque no hay reposo absoluto, desde el momento en el que hemos elegido un sistema de referencia, con este gesto lo hemos sacado del movimiento.

Supongamos que, al igual que su compañero de la Tierra, un físico instala el aparato Michelson-Morley en el Sol. El físico de la Tierra, si elige la Tierra como sistema de referencia, y por esta decisión la fija en reposo, puede desarrollar su experimento sin ninguna dificultad. Pero cuando se entera de que el físico que está en el Sol mide la misma velocidad de luz, interpretará este resultado con la ayuda de los cálculos de transformación de Lorentz. Dicho de otra manera, según él, el físico que está en el Sol, que se encuentra en movimiento, ha llegado al mismo resultado porque, después del movimiento de la luz en la dirección del desplazamiento del sistema, se ha producido un achicamiento de la distancia recorrida, es decir según los cálculos de Lorentz la longitud $l$ del brazo del aparato será $l \sqrt{1-\frac{v^{2}}{c^{2}}}$ donde $v$ representa la velocidad del Sol en relación con la Tierra, y $c$ es la velocidad de la luz. Dado que $v$ necesariamente es más pequeño que el $c$ que no puede ser superado y prácticamente tampoco puede ser tocado por otro movi- 
miento, la relación $\frac{v^{2}}{c^{2}}$ está por debajo de $l$, y por eso el resultado de la fórmula será más bajo que el multiplicado $l$. También, donde el reloj del Sol ha medido $t$ segundos, en realidad han pasado solamente $\frac{t^{\prime}}{\sqrt{1-\frac{v^{2}}{c^{2}}}}$ segundos, por lo tanto el tiempo se ha dilatado. Finalmente,

si los relojes situados en el Sol a distancias iguales $l$ en la dirección del movimiento de éste mostrarán la misma hora para el físico de allí, que no es consciente de su movimiento, el físico de la Tierra debe tener en cuenta que al arreglar estos relojes, las señales que circulaban entre ellos no han recorrido el mismo camino allí y allá y por eso en el lugar donde estos relojes muestran simultaneidades para el físico del Sol, en

realidad se trata de instantes sucesivos a una distancia de $\frac{l v}{c^{2}}$ segundos, según el reloj del físico del Sol y $\frac{l v}{c^{2} \sqrt{1-\frac{v^{2}}{c^{2}}}}$ segundos según el reloj del físico de la Tierra: por lo tanto las simultaneidades se alejan.

Sólo que también el físico del Sol afirmaría lo mismo sobre las mediciones del compañero de la Tierra. Se consideraría igual de inmóvil y acordaría el calificativo correcto a los datos medidos en su propio sistema, y los datos del físico de la Tierra irían a ser corregidos por las fórmulas de Lorentz. Así tenemos dos representaciones matemáticas del universo que son diferentes en cuanto a las cifras que figuran en ellas, pero corresponden en cuanto a las relaciones internas de los fenómenos descritos, es decir, en cuanto a «las leyes de la naturaleza». Y si imaginamos no solamente dos, sino $n$ sistemas, todos los físicos actuarían de la misma forma, tomando en cuenta a los demás en función de su velocidad en relación con el propio sistema, y corregirían sus datos según las diferencias de velocidad. Por consiguiente constatarían $n$ tiempos según el número de sistemas.

El filósofo del que habla Bergson no se conforma con los cálculos de Lorentz en cuanto a la diferencia entre los tiempos, sino que distingue entre la duración real del tiempo medido, la simultaneidad percibida de la simultaneidad indicada por los relojes, es decir, el tiempo 
que pasa del tiempo pasado, y evalúa los tiempos multiplicados de la física en base a estas diferencias.

La duración es el tiempo interior, percibido de manera no mediada, una continuidad que no contiene una cosa cualquiera que continúa, y tampoco estados por los cuales pasa: «cette transition, seule naturellement experimentée, est la durée même» (Bergson [1922] 1923: 54-55). La condición indispensable de este tiempo interior es la memoria que ejerce su efecto en transformación a través de la percepción del momento precedente en el momento que le sucede a éste, es decir, no permite la descomposición de lo que hubo antes y lo que será después en puntos cerrados en sí que aparecen y desaparecen en nuevos y nuevos momentos del presente. En la concepción de Bergson el tiempo significa para nosotros, ante todo, esta transición sin interrupción, es decir, una multiplicidad indivisible, una sucesión sin interrupción, que a lo que más se parece es a la música. El tiempo de las cosas «duraderas» es considerado secundario, relacionado con la transición permanente de la conciencia por percepción: «A chaque moment de notre vie intérieure correspond ainsi un moment de notre corps, et de toute la matière environnante, qui lui serait "simultané": cette matière semble alors participer de notre durée consciente» (Bergson [1922] 1923... 55-56). Este «participer» (participación) se refiere al hecho de que la materia misma, al pertenecer al ámbito de la experiencia, es vivida en nuestra duración interior. El tiempo interior está relacionado con la conciencia en la que participan también las cosas percibidas que son consideradas duraderas.

La duración es indivisible porque cualquier momento marcado que intentaría dividirla en períodos, al mismo tiempo detendría también su paso por esta fijación, y de este modo la duración no podría seguir siendo lo que es: una transición sin interrupción. Y si es indivisible, en consecuencia tampoco se puede medir, dado que medir significa división y superposición, sin embargo las duraciones estrictamente sucesivas no se pueden sobreponer en vista de su comparación. Los períodos en los que la duración es dividida, en realidad no son partes de la duración, sino secciones del espacio en las que la huella de la duración puede ser marcada. Aunque, mientras se mide, las secciones de esta línea son identificadas con las unidades de la duración, nunca se puede dividir el desarrollo indivisible, sino solamente el tiempo pasado. La primera es el movimiento puro en sí, y la última es una trayectoria unida al movimiento como una representación espacial, es decir un símbolo de ésta. Sin un desarrollo continuo no sería más que un espacio que nunca más pudiera representar el tiempo. 
El paso de la duración que no se puede medir del desarrollo al tiempo pasado mesurable significa añadir una nueva dimensión al espacio dado, independientemente de si éste es un punto o un espacio uni-, dio tridimensional. Sólo una nueva dimensión hace posible que la multiplicidad indivisible de la sucesión se convierta en una fila, es decir, una multiplicidad divisible. En un espacio punto la duración significa un cambio cualitativo infinito de este punto. Pero si la velocidad de esta sucesión es considerada infinita, entonces los puntos sucesivos se van a dar simultáneamente, es decir, se van a situar en una línea. Pero, a partir de este momento no estamos ante un punto, sino ante un espacio unidimensional. Vamos a actuar de la misma manera con una sucesión cualitativa de una línea, si con añadir la segunda dimensión, creamos un espacio plano, o si transformando la sucesión de las imágenes planas por una velocidad de cambio infinita, creamos una superposición tridimensional.

Una vez que en nuestro mundo tridimensional fijamos el tiempo pasado y lo calculamos sólo por simultaneidades, sin tener acceso a la duración de los intervalos, no hacemos otra cosa que añadir a nuestro espacio una cuarta dimensión virtual. En el espacio obtenido de esta manera el pasado, el presente y el futuro se suceden en una fila. Esta espacialización en física se debe a que no podemos percibir el tiempo por medio de las matemáticas, podemos solamente sustituirlo por simultaneidades que se pueden contar. Estos momentos contables ya no tienen duración, son símbolos creados por nuestra mente que marcan el movimiento incesante de la sucesión temporal por detenimientos virtuales con la ayuda de los puntos prestados del espacio. Esta espacialización puede ser llamada tiempo porque, gracias a la percepción, somos capaces en cualquier momento de volver de la trayectoria dividida en secuencias desarrolladas a la sucesión del desarrollo. En estas ocasiones la simultaneidad de los momentos es cambiada por flujos simultáneos.

La simultaneidad de los momentos difiere de la simultaneidad de los flujos. El flujo se refiere siempre a la duración interior. «Nous appelons alors simultanés deux flux extérieurs qui occupent la même durée parce qu'ils tiennent l'un et l'autre de la durée d'un troisième, le nôtre» (Bergson, [1922] 1923: 68). El vuelo de un pájaro y el paso de las nubes son percibidos conforme al flujo incesante de la vida interior, independientemente de si nuestra atención se orienta hacia los tres en una sola percepción, o está dividida entre exterior e interior. En todo caso, el vuelo y el paso están incluidos en nuestra duración, y esto constituye para nosotros la simultaneidad primordial o natural. 
La simultaneidad de los momentos se relaciona con el tiempo espacializado, es decir, de medición. Sólo a través de ella es posible registrar la simultaneidad entre un acontecimiento exterior y una posición cualquiera del índice del reloj, y percibir «les simultaneités de ces moments avec des moments de notre durée» (Bergson, [1922] 1923: 70), es decir mirar el valor de esta medición como una medida de tiempo.

La física (o la ciencia, en general) opera en exclusividad con datos de medición y, en el caso del tiempo, esto significa contar los momentos y la constatación de las simultaneidades. Pero los intervalos medidos muestran a la ciencia solamente un punto de las dos cabezas, por consiguiente lo que dura o pasa en estos intervalos se escapa a la medición y al contar. Si los movimientos del mundo (incluidos los utilizados para medir el tiempo, es decir el movimiento de los relojes) se dieran prisa o si fueran despacio de repente, para la ciencia nada cambiaría. Sólo para la percepción significaría un cambio, porque la percepción es capaz de comparar la duración de las cosas con el flujo de la vida interior.

Bergson no hace la distinción entre «los tiempos» de la misma manera que el físico, sino que nos llama la atención sobre la diferencia entre el tiempo percibido en una experiencia y el tiempo convencional-simbólico. En su proyecto de acercamiento filosófico a la teoría de la relatividad, intenta por medio de esta distinción resolver la confusión que se debe a las tentativas de «avanzar» a la física al rango de metafísica. Las paradojas no resultan del hecho de que el tiempo se simboliza por una línea, sino del error de designar esta línea utilizando el término «tiempo», incluso cuando la puesta en fila de sus puntos ya no puede ser transformada en sucesión por medio de una percepción. O, si utilizamos (porque resulta más cómodo) también en estas situaciones la palabra "tiempo", no debemos olvidar que ésta es totalmente arbitraria, pura convención, absolutamente independiente del tiempo que percibimos en una experiencia.

$\mathrm{Si}$, utilizando estas consideraciones, reexaminamos la multiplicación del tiempo en la teoría de la relatividad, llegamos a conclusiones diferentes de las obtenidas por el físico.

Tengamos en cuenta dos sistemas que se mueven, el uno dependiendo del otro, a una velocidad constante sobre una línea recta y los nombramos $R$ y respectivamente $R$ '. Un físico mide en los dos sistemas el tiempo del desplazamiento del rayo de luz de la pantalla de vidrio hasta el espejo y de vuelta, sobre aquel brazo del aparato Michelson-Morley 
que apunta en la dirección del movimiento. El tiempo leído por el físico del sistema $R$ en su reloj del mismo sistema es un tiempo vivido, y en este sentido real. Porque en el intervalo entre el ir y volver del rayo, el físico ha percibido una duración, y el movimiento de los indicadores del reloj es «simultáneo» con este flujo interior, es decir, participa en éste, y por consiguiente, le puede servir como medida. El físico del sistema $R^{\prime}$ que, al igual que su colega, elige su propio sistema de referencia, también cuenta un tiempo vivido, por tanto real. Según la teoría de la relatividad, yendo despacio o velozmente, la multiplicación del tiempo depende exclusivamente de la velocidad de un sistema en relación con el otro, por consiguiente podemos suponer que $R^{\prime}$ es una copia de $R$. Así el tiempo medido por los dos físicos es el mismo porque, una vez elegidos como sistemas de referencia, los dos sistemas no se mueven; y teniendo, a causa de su inmovilidad, la misma velocidad, se pueden sustituir el uno al otro. De esto resulta que el tiempo vivido y registrado en el interior de los dos sistemas no sólo que es real de la misma manera, sino que, pura y simplemente es idéntico. ¿Cuál es entonces el tiempo que difiere de éste? El que el físico del sistema $R$ atribuye al físico del sistema R', y que es más lento en relación con el propio sistema. Este tiempo atribuido no es vivido por ninguno de los físicos, además tampoco puede ser pensado como un tiempo vivido por alguien. Porque aunque los físicos tratan aquel tiempo que pasa más despacio, bajo la etiqueta del otro, actuando de esta manera no piensan en el otro como en un ser que percibe. Si ellos imaginaran al otro como a alguien que vive y mide su propia duración, por medio de esta imaginación cada uno se alejaría de su propio sistema, y se trasladaría al sistema del otro. Y esta trasferencia significaría que cada uno se considera una visión del otro. A lo largo de los experimentos sin embargo, pasa lo contrario: cuando el físico de $R$ le atribuye un tiempo más lento al sistema $R^{\prime}$, deja de ver en su colega de allí un ser que percibe, la física no está interesada en la percepción de éste, sino se hace la siguiente pregunta: ¿cómo se pueden integrar los datos de medida del colega en la descripción del universo desde la perspectiva del propio sistema? Éste es el objetivo de las transformaciones Lorentz. Por lo tanto, el tiempo atribuido al otro no es otra cosa que una expresión matemática, es decir, un símbolo para la designación del hecho de que, esta vez, hemos elegido a $R$ como sistema de referencia y no a $R^{\prime}$. Si tenemos que examinar un número facultativo de sistemas, de los cuales establecemos uno como sistema de referencia, entonces por medio del cálculo de los tiempos más o menos lentos, es decir, en una representación matemática del mundo, expresamos la relación de cada sistema con la velocidad nula del fijado. 
De esta manera, en sentido filosófico, no se trata de la multiplicación del tiempo, dado que para nosotros sólo el tiempo vivido es real, el que coincide cada vez con el tiempo del sistema de referencia. Todos los demás «tiempos» no tienen duración; son tiempos en los que no es posible una sucesión de acontecimientos, el deterioro de las cosas o el envejecimiento de la gente. A pesar de que estos tiempos pueden ser útiles a la hora de expresar la perspectiva matemática, respectivamente física, no son otra cosa que unas convenciones en cuya denominación se ha mantenido el término «tiempo» referente (también) a la duración vivida.

Los físicos que están en sus sistemas con velocidades distintas no comparten el mismo punto de vista, pero precisamente esta diferencia les une como físicos. Mantienen estrictamente su propia perspectiva, porque han llegado al acuerdo de que la representación matemática del universo es posible solamente desde un punto fijo con el que se relacionan todos los demás, de forma que las leyes de la electromagnética sean idénticas para cada posición. Cada físico procedería de la misma forma que cualquiera de sus compañeros, es decir, mediría de la misma manera, y calcularía igual las simultaneidades medidas, porque todos se inscriben en la misma física.

Sin embargo, el filósofo de Bergson no puede ser sustituido por el físico. No porque no se encuentre en una relación recíproca, sino porque esta reciprocidad no es simétrica como en el caso de los físicos. La espacialización del tiempo tampoco le es ajena al filósofo, porque «la recette en est déposée dans le langage» (Bergson, [1922] 1923: 70). Si no espacializaramos el tiempo, no sólo que no lo podríamos medir sino que tampoco podríamos hablar de él. El filósofo, sin embargo, es capaz de distinguir el tiempo real del espacializado porque no mide sólo contando, sino cualitativamente; «teniendo en cuenta» no sólo los datos mesurables, sino también la duración indivisible y no-mesurable. El físico, precisamente por ser físico, excluye todo lo que no es mesurable para obtener expresiones matemáticas de las leyes constantes. Y el físico percibe, y por consiguiente vive, una duración por medio de su sistema de referencia, pero registra sólo los datos medidos y las simultaneidades de los momentos de éstos. Así, la duración para él se convierte en equivalente de los tiempos calculados, no vividos por nadie, atribuidos a los sistemas representados recurriendo a la perspectiva. La teoría de la relatividad considera el tiempo vivido o real sólo como una instancia posible de los virtuales, dado que para el físico todos son datos del tiempo medido. Por lo tanto, la multiplicación del tiempo se basa en una identificación convencional, y el tiempo único real del filósofo se 
basa en una diferencia entre este convencional-simbólico y duración. Aquí está la reciprocidad asimétrica: las dos prácticas, aunque se pueden relacionar, no pueden ser sustituirse una por otra. En otras palabras, la física de Einstein y la filosofía de Bergson resultan igual de posibles, pero no se pueden deducir la una de la otra. Einstein cuenta, es decir, detiene la transición continua de la percepción por datos de medición; Bergson, sin embargo, mide la duración de lo medido con la percepción. El primero hace cálculos matemáticos, el segundo sigue flujos que se pueden vivir. «El tiempo multiplicado» y «el único tiempo real» son una duración propia del desarrollo de Einstein o de Bergson, respectivamente, y no dependen de la velocidad de un sistema físico cualquiera, ni de un punto de vista teórico, sino de la práctica que ritma el medir, el contar o el flujo de la percepción. Por consiguiente, el tiempo no puede ser una cuarta dimensión del espacio físico y tampoco una continuidad matemática separada por este espacio, sino la duración de algo que se puede llamar física, ofilosofía en un sentido etológico, indiferente a las particularidades disciplinarias.

Bergson se ha visto impedido en la investigación del carácter etológico del tiempo-duración por el hecho de que, gracias a la presión ejercitada por el propio sistema de pensamiento, al igual que gracias a la epistemología positivista, ha separado lo percibido de su percepción. Sobre la relación gnoseológica entre estos polos, Bergson ha podido identificar sólo dos maneras de comportamiento: la práctica del físico, es decir la ciencia, y la práctica del filósofo, es decir la metafísica. La primera trata de llegar a la multiplicidad del movimiento y a la equivalencia entre las constataciones y los símbolos estables, y la última, todo lo contrario, se adapta al movimiento a través de la intuición, para llegar luego a constataciones «conceptualmente apenas expresables». Incluso en estas dos prácticas distintas, Bergson atribuye tiempo real sólo a la percepción, a la que considera única sucesión verdaderamente común al filósofo y al físico. Al mismo tiempo, separando la continuidad externa de las cosas del flujo interior de la conciencia, mantiene la unidad de la duración a través de la afirmación que los movimientos externos participan en el flujo interior. Esta expresión platónica oscura esconde una aporía sin resolver, al igual que la filosofía del antiguo pensador:

Il n'est pas douteux (...) que notre perception fasse partie de notre conscience, ni qu'il entre quelque chose de notre corps, et de la matière qui nous environne, dans notre perception: ainsi, notre durée et une certaine participation sentie, vécue, de notre entourage matériel à cette durée intérieure sont des faits d'expérience. Mais (...) la nature de cette participation 
est inconnue: elle pourrait tenir à une propriété qu'auraient les choses extérieures, sans durer elles- mêmes, de se manifester dans notre durée en tant qu'elles agissent sur nous et de scander ou de jalonner ainsi le cours de notre vie consciente.

(Bergson, [1922] 1923: 57)

La participación es «de naturaleza desconocida» porque, en su investigación, Bergson retrocede en cuanto a su propio radicalismo. Aunque reconoce que las cosas no pueden durar temporalmente, se niega a aceptar lo que resulta de esta afirmación: que las cosas son puros símbolos, que solamente el filósofo les atribuye duración real, por lo tanto tienen la misma naturaleza que los tiempos ficticios del físico. Dado a que Bergson trata a cualquier precio de otorgarle al «ámbito material» cualidades objetivas, independientes de nuestras prácticas, y al mismo tiempo sabe que este deseo es contradictorio, formula sus hipótesis con una conciencia poco clara:

A supposer que cet entourage «dure», rien ne prouve rigoureusement que
nous retrouvions la même durée quand nous changeons d'entourage: des
durées différentes, je veux dire diversement rythmées, pourraient coexister.
Nous avons fait jadis une hypothèse de ce genre en ce qui concerne les
espèces vivantes. Nous distinguions des durées à tensión plus ou moins
haute, caractéristiques des divers degrés de conscience, qui s'échelonne-
raient le long du règne animal. Toutefois nous n'apercevions alors, nous
ne voyons, encore aujourd' hui, aucune raison d'étendre à l'univers maté-
riel cette hypothèse d'une multiplicité de durées. Nous avions laissé ouver-
te la question de savoir si l' univers était divisible ou non en mondes indé-
pendents les uns des autres; notre monde a nous, avec l'élan particulier
qu'y manifeste la vie, nous suffisait. Mais s'il fallait trancher la question,
nous opterions, dans l'état actuel de nos connaissances, pour l' hypothèse
d'un Temps matériel et universel. Ce n'est qu' une hypothèse, mais elle est
fondée sur un raisonnement par analogie qui nous aura rien offert de plus
satisfaisant.

(Bergson, [1922] 1923: 57-58)

Trataremos de ofrecer una resolución más aceptable. Después de las investigaciones fenomenológicas, hermenéuticas y desconstructivistas, es decir, el redescubrimiento de las obras de Nietzsche y Freud por los adeptos de estas corrientes, nos encontramos en una situación mucho más ventajosa que Bergson, por lo tanto no deseamos para nada considerar lo que viene a continuación como una demostración de una genialidad vencedora. De hecho, se trata de seguir una dirección de investigación que no sólo que ha sido iniciada por el autor de la obra Durée et simultanéité, sino incluso preparada, más o menos conscientemente, para ser continuada. 
El sostener la realidad del mundo material en las investigaciones de Bergson conduce a una aporía que, al mismo tiempo, puede ser también muy productiva. Si alguna vez, de modo hipotético, hemos atribuido duración a las cosas y a los fenómenos que nos rodean, ¿por qué no podrían tener éstos duraciones distintas? Pero eso, seguramente, pondría en peligro la singularidad del universo por multiplicación. Se le puede reprochar a Bergson que, en cuanto a este problema, no ha pensado en términos temporales. La multiplicación del ámbito material durable es concebida por el filósofo como una división del universo en mundos separados, es decir, de manera completamente espacial, lo que es una idea anacrónica, pre-bergsoniana. Resulta increíble que ni siquiera aparezca en este libro la posibilidad de seguir el flujo cualitativo de estos mundos, consultar «el mundo»como un movimiento cambiante. ¿O quizá haya aparecido sin embargo, pero ha sido prohibida de inmediato, porque parecía poner en peligro la concepción completa de duración? Porque el flujo de la vida interior puede convertirse en una sucesión pura, es decir memoria que mantiene el precedente en lo ulterior solamente por falta de objeto. Incluso en la melodía escuchada con los ojos cerrados «il faudrait effacer d'abord la différence entre les sons, puis abolir les caractères distinctifs du son lui-même [...] pour retrouver enfin le temps fondamental» (Bergson, [1922] 1923: 55). En otras palabras, la duración se define en relación con las cosas sin conciencia, concebida con esbeltez en contraste con la rigidez y la espacialidad de las cosas. Al igual que los símbolos cobran cuerpo en la inmovilidad en la semejanza de las cosas.

Aunque el capítulo titulado De la nature du temps empieza así: «Il n'est pas douteux que le temps ne se confonde d'abord pour nous avec la continuité de notre vie intérieure» (Bergson [1922] 1923: 54), a lo largo de toda la obra se mantiene el dualismo clásico interior / exterior. $\mathrm{El}$ investigador del tiempo descubre el impulso interno del movimiento, pero no tiene el valor de entregarse a él, sino que mantiene la prioridad natural del mundo material, intenta, incluso a pesar de la contradicción, relacionar todo con esta referencia. En realidad la duración se mide con cosas, como si fuera mesurable. Por lo tanto, la realidad no se reduce al tiempo, como lo esperaríamos en el caso de la consecuencia bergsoniana, es decir, cosas que sin ser tiempo son sin embargo realidad. Si nuestra única experiencia real es la duración, y los símbolos con los que dividimos el flujo de ésta en estructuras espaciales, son imaginarios, ficticios o virtuales, entonces es problemática la introducción de una tercera categoría, que debe participar en la duración para poder adquirir una naturaleza temporal y como consecuen- 
cia experiencia real, porque esta participación es impedida por la suposición tácita de que ante todo, el tiempo debe participar en una realidad perteneciente de manera a priori al mundo material. Bergson trata de escapar de esta paradoja cada vez que se esconde en el mundo suficiente y completo ${ }^{2}$, formado sólo por la duración y sus símbolos. Él se atreve a hacer esta mutación sólo metodológicamente, es decir, tapada con metodología: «Nous ne trancherons pas la question de savoir si toute réalité possède ce caractère (que es perceptible). Il ne s'agira ici de la réalité du temps» (Bergson, [1922] 1923: 90). El deseo rechazado de escapar a la prioridad del mundo material se manifiesta a veces en la estilística de las proposiciones: «Fort heureusement nous n'avons à nous occuper, dans tout ce qui va suivre, que d'une seule réalité» (Bergson, [1922] 1923: 87).

Sin embargo, de alguna manera la realidad del mundo material, «los flujos exteriores» supuestos, pueden cobrar mayor importancia a la hora de abordar la problemática del tiempo, porque, aunque de manera misteriosa, participan en el flujo de la percepción, al mismo tiempo, de una manera no menos misteriosa, sobrepasan la percepción. Por su culpa - aunque sólo por un instante - se plantea el problema de la multiplicidad de lo que se encuentra fuera de la percepción, es decir, la posibilidad de los diferentes ritmos de duración. Parece que este enigma bergsoniano es la proyección de una sospecha rechazada. La insinuación de la multiplicidad, basada en una «relatividad» no-física del tiempo, se ve impedida en su clasificación y formulación por una exigencia científica. En cuanto a ésta, voy a citar de nuevo el pasaje sobre la separación metodológica de la realidad temporal:

Fort heureusement nous n' avons à occuper, dans tout ce qui va suivre, que d'une seule réalité, le temps. Dans ces conditions, il nous sera facile de suivre la règle que nous nous sommes imposée dans le présent essai: celle de ne rien avancer qui ne puisse être accepté par n'importe quel philosophe, n'importe quel savant,- rien même qui ne soit impliqué dans toute philosophie et dans toute science.

(Bergson, [1922] 1923: 87-88).

El reconocimiento del carácter etológico del tiempo se ve impedido por esta estricta exclusión y sus consecuencias. La coordinación (coincidencia) de la realidad del tiempo con la regularidad lógica común a

2 Ante todo falto de los problemas sin resolver. 
la ciencia y a la filosofía, reduce el flujo de la duración a una sola práctica. Lo que se proyecta en el discurso de Bergson como opción para la realidad que va a ser investigada, en realidad es la reducción del tiempo al horizonte de la percepción. Y la idea presupuesta pero no admitida de las múltiples duraciones, mantenidas sólo como entre paréntesis, en realidad es la proyección de la hipótesis rechazada sobre «la naturaleza de la temporalidad».

Bergson habla, muchas veces, del mundo «de nuestra vida interior», del «gruñido incesante» de los pasos continuos, pero si examinamos a fondo sus explicaciones, descubrimos que ellas se limitan a un solo aspecto de este gruñido: sólo a la duración de la percepción. Cualquier cambio cualitativo es interpretado a través de una psicología elementar que se somete a la lógica de observación de las cosas, aún cuando se acentúa la exclusión de los objetos y de los estados subjetivos de la duración pura. Esta psicología, aunque, según toda la teoría de Bergson, lleva dentro la posibilidad de la multiplicidad del tiempo nofísico, es más bien el proceso mental de observación de las cosas purificado por la abstracción, que una duración de la vida vivida conteniendo también las posibles alternancias de la percepción. Al abordar este «proceso primario» Bergson pasa por alto las cosas (realidades que tienen el mismo rango que el tiempo) en base a unas consideraciones metodológicas. La mejor vía, la más «satisfactoria», la podemos encontrar, si no proyectamos estas realidades diferentes en las cosas del mundo material, para excluirlas luego de la duración por un acto típico de la percepción, es decir, por abstracción, sino que aceptamos el carácter temporal de «las demás realidades» y empezamos a investigar las prácticas que siguen un ritmo de duración distinto del ritmo de la percepción.

La teoría de Bergson distingue variantes en la jerarquía dentro de la misma duración, definiéndolas como peldaños de la conciencia «en la escalera de la evolución». ¿Será justo llamar estos peldaños «duraciones con ritmos diferentes»? En la medida en que se trata solamente de peldaños, es decir, proporciones en las que ciertas especies, situadas en distintas posiciones en la jerarquía evolucionista, participan en la percepción humana; sería más justo hablar de distintos tempo de un solo ritmo. Bergson constata diferencias cuantitativas entre duraciones de «más o menos alta tensión» dentro de la misma percepción. Por estas «frecuencias» no se cambia el ritmo de la duración, porque conforme con esta concepción influyen sólo en la medida de la intensidad del esfuerzo mental o la capacidad espiritual. La hipótesis de las frecuen- 
cias de percepción tiene en cuenta, de nuevo, la realidad del mundo material, y la acrobacia «analógica» de su formulación nos muestra el origen de la idea de unidad del tiempo que «es espontánea y obscura para la mente sencilla, y se convierte en consciente y clara sólo en la percepción filosófica». De nuevo estamos ante unos peldaños que relacionan las percepciones en distintos niveles de la abstracción, según viene a continuación:

Toutes les consciences humaines sont de même nature, perçoivent de la
même manière, marchent en quelque sorte du même pas et vivent la même
durée. Or, rien ne nous empèche d' imaginer autant de consciences humai-
nes qu'on voudra, disséminées de loin en loin à travers la totalité de l'u-
nivers, mais juste assez rapprochées les unes des autres pour que deux
d'entre elles consécutives, prises au hasard, aient en commun la portion
extrème du champ de leur expérience extérieure. Chacune de ces deux
expériences extérieures participe a la durée de chacune des deux cons-
ciences. Et puisque les deux consciences ont le même rythme et durée, il
doit être ainsi des deux expériences. Mais les deux expériences ont une
partie commune. Par ce trait d'union, alors, elles se rejoignent en une
expérience unique, se déroulant dans une durée unique qui sera, à volon-
té, celle de l'une de l'autre des deux consciences. Le même raisonnement
pouvant se répéter de proche en proche, une même durée va ramasser le
long de sa route les événements de la totalité du monde matériel; et nous
pourrons alors éliminer les consciences humaines que nous avions d'abord
disposées de loin comme autant de relais pour le mouvement de notre pen-
sée: il n'y yura plus que le temps impersonnel ou s'écouleront toutes coses.

(Bergson, [1922 [ 1923: 58-59).

Esta deducción lógica muy regular y totalizadora nos muestra los límites de la concepción de Bergson sobre el tiempo. Parte de la suposición de que no hay tiempo real (vivido) fuera de la percepción. Cualquier práctica humana o de un animal se basa de este modo en la duración de la percepción, es decir en un solo ritmo que permite posiblemente tiempos distintos. El argumento principal es esta hipótesis compartida por cada «experto» (sostenida entre otras por la teoría de la relatividad) en una medida más o menos consciente, que hace posible la percepción del otro y de las cosas materiales. En un universo en el que sólo la percepción es real, es decir, lo que participa en la percepción en forma de experiencia, no puede existir un ritmo diferente al ritmo de la percepción. De este modo el universo se descompondría en mundos independientes entre los cuales dejaría de existir cualquier relación. Bergson comprende la naturaleza del tiempo como un principio unitario y absolutamente estable, excluyendo de esta manera, desde un principio, la posibilidad del cambio del ritmo de duración. Supongamos que su lógica admite la misma duración en el caso de 
El SIMBOLISMO DE LA TEORÍA DE LA RELATIVIDAD Y LA DURACIÓN COMO...

cualquier número de personas, pero que no acepta que la vida interior de un solo hombre pueda significar duraciones con ritmos diferentes. Sin embargo, sustituyendo la naturaleza duradera, que no cambia con la transición permanente del comportamiento, vamos a obtener la multiplicidad imprevisible de las duraciones, asequible por la resonancia de nuestros gestos provocada por sus ritmos. Por supuesto, la resonancia no se realiza con apretar un botón, sino por medio de ejercicios de comportamiento, es decir, a través de ritos. 